\title{
Application of Artificial Immune System in Structural Health Monitoring
}

\author{
Jiachen Zhang and Zhikun Hou \\ Mechanical Engineering Department, Worcester Polytechnic Institute, 100 Institute Road, Worcester, MA 01609, USA \\ Correspondence should be addressed to Jiachen Zhang; jzhang2@wpi.edu
}

Received 30 November 2013; Accepted 24 July 2014; Published 20 August 2014

Academic Editor: Greg Foliente

Copyright (c) 2014 J. Zhang and Z. Hou. This is an open access article distributed under the Creative Commons Attribution License, which permits unrestricted use, distribution, and reproduction in any medium, provided the original work is properly cited.

\begin{abstract}
A large number of methods have been proposed in the area of structural health monitoring (SHM). However, many of them rely on the prior knowledge of structural-parameter-values or the assumption that the structural-parameter-values do not change without damage. This dependence on specific parameter values limits these methods' applicability. This paper proposes an artificial immune system- (AIS-) based approach for the civil structural health monitoring, which does not require specific parameter values to work. A linear three-floor structure model and a number of single-damage scenarios were used to evaluate the proposed method's performance. The high success rate showed this approach's great potential for the SHM tasks. This approach has merits of less dependence on the structural-parameter-values and low demand on the training conditions.
\end{abstract}

\section{Introduction}

Structural health monitoring (SHM) refers to the process of implementing a strategy to identify the damage in engineering infrastructures. The damage here can be changes to the material and/or geometric properties, boundary conditions, and system connectivity [1]. The specific objectives of SHM systems are to acquire the information about the damage existence, the damage location, the damage type, and the damage severity [2]. An effective SHM system could detect the structural damage in its early stage, well before a catastrophic structural failure, which threatens the safety of people's life and property. Also, SHM systems provide valuable information for postevent damage assessments and help to develop a condition-based repair priority. Various schemes have been developed to extract the structural health information from the measured structural dynamic response. These techniques include, but are not limited to, modal-based system identification techniques, wavelet-based approaches, neural network-based schemes, Kalman filter methods, statistical approach, and computer-based machine learning techniques [3].

As one of the machine learning techniques, the recently developed artificial immune system (AIS) is an interdisciplinary area, which relates to immunology, computer science, and engineering [4]. The AIS learns from the principles and mechanisms in the biological immune system (BIS) and takes advantage of the tremendous computation power of modern computers. Many algorithms of the AIS have been developed, such as the negative selection algorithm [5], the positive selection algorithm [6], and the clonal selection algorithm [7]. These methods have been successfully applied to many engineering areas and show great potential to be applied in the SHM task.

Chen and Zang $[8,9]$ proposed to apply the artificial immune pattern recognition to perform a task of structure damage classification. They employed the principal component analysis (PCA) and multiple regression analysis to extract feature vectors from the structural vibration response. Their method was evaluated using two structures: (1) the benchmark structure proposed by the IASC-ASCE (International Association for Structural Control-American Society of Civil Engineers) Structural Health Monitoring Task Group and (2) the three-story frame provided by Los Alamos National Laboratory. Their results showed that the proposed method is suitable for the structure damage classification.

Xiao [10] explored the feasibility of using the natural frequencies and mode shapes of structure's vibration response as feature vectors for the AIS. Damage was detected, located, and estimated by the well-trained antibodies. Both of the 
natural frequencies and mode shapes exhibit different behavior under different damage conditions. Analytical calculation results and numerical simulation results were compared. The proposed method was tested by the simulation data and in a seismic scenario. Satisfactory results were acquired.

Construction of an AIS requires a training stage. These previous works have a common limitation: their training processes heavily rely on specific structural parameter values (SPVs). Either the training needs data from the interested real world structure or it requires an accurate model for simulation. If the data from the interested real world structure is required, each structure will need an AIS uniquely trained for it to perform the SHM task. The AIS also needs to be retrained if any SPV (like the mass) of the interested structures changes, which is not uncommon for some structures like the offshore oil platforms. Alternatively, training the AIS using simulation requires exact structure model. Unfortunately, exact structure model is unobtainable in most occasions. Differences between the parameters of the model used for simulation and the ones of the real structure will deteriorate the method's performance and limit its applicability to real world problems. Therefore, the dependence of the AIS on specific SPVs significantly limits its applicability to the practical SHM area.

In this paper, a SPV-insensitive AIS is proposed and applied to the SHM. The proposed AIS is applicable to structures having similar but different SPVs with the structure used for training. Also, it allows the system to be trained using a model with errors but still exhibits satisfactory performance.

This paper is structured as follows. Section 2 explains the proposed methodology and states the procedure to construct the AIS. Section 3 demonstrates the methodology using an example structure and also evaluates its performance. Section 4 concludes the presented work and Section 5 points out unsolved issues and future research directions.

\section{Methodology}

2.1. Introduction to the Artificial Immune System (AIS). Our immune systems protect us from the external invaders to our bodies, for example, the pathogenic organisms. The immune system initiates a primary-immune-response when antigens, that is, foreign substances that invade our body, are detected. The immune cells having high affinities with this antigen undergo clone and mutation processes to generate more immune cells with similar antibodies, which are specialized proteins that can neutralize some specific antigens. The generated antibodies react with this invading antigen and prevent it from harming the body. After this antigen has been neutralized, some immune cells become memory cells and stay in our immune system. If the same kind of antigen intrudes our body again, these memory cells will initiate a secondary-immune-response to fight these invaders. Readers are referred to [11] for a more detailed discussion about the biological immune system (BIS).

From the SHM's perspective, one attractive feature of the BIS is its capacity of learning from its experiences to improve

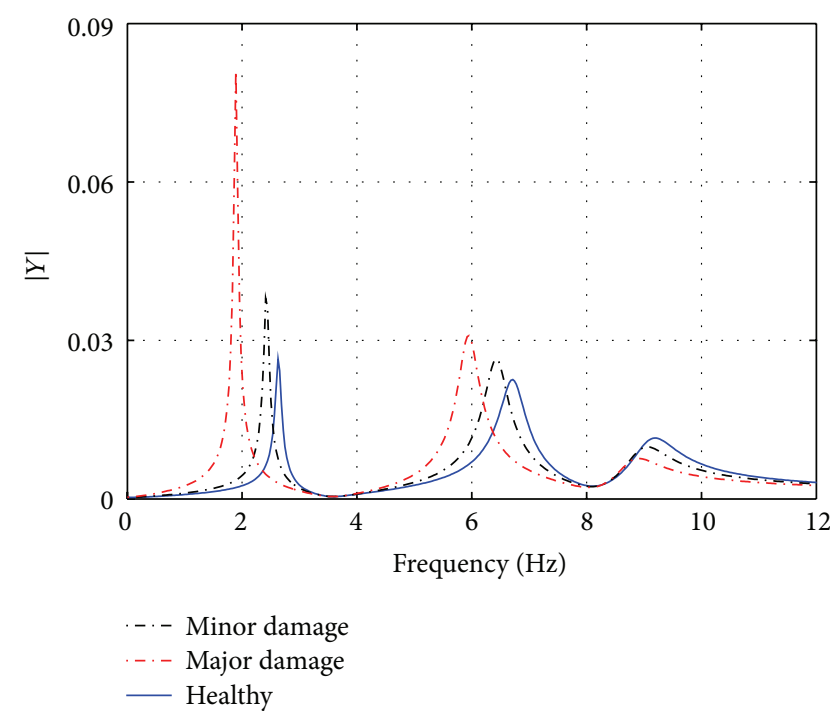

FIGURE 1: An example of changes in the structural natural frequencies caused by different damage severities.

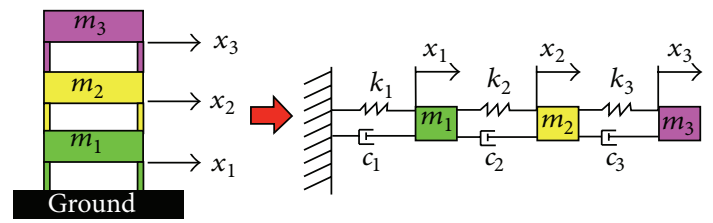

FIgURE 2: A schematic of the three-story building and its equivalent three-degree-of-freedom mass-spring-damper model.

its own effectiveness and efficiency. One AIS is proposed here to apply this attractive feature to the SHM, which takes advantages of both the BIS' learning capability and the modern computer's computational power. The proposed AIS is based on the methodology proposed by Chen and Zang $[8,9]$ and is minutely discussed in Section 2.3.

Because many concepts and mechanisms in the AIS and the BIS share the same name, Table 1 presents a comparison between the meanings of the same name in the two areas to clarify their differences and emphasizes their links.

2.2. Damage-Location-Sensitive Feature Vector. Frequencies, as one of the modal parameters, are functions of the physical properties (such as the mass and the stiffness) of the structure. Therefore, changes in the physical properties, as a result of damage, will cause changes in the frequencies [12]. This dependence of frequencies on physical properties makes frequency an indicator of structural-health-condition. An example of the frequency changes caused by damage is shown in Figure 1.

Figure 1 is the Fourier spectrum generated from the simulated free vibration data of a linear three-degree-offreedom (3DOF) structure model (Figure 2). The "minor damage" and "major damage" in Figure 1 were defined as the conditions where the first floor loses $25 \%$ of its stiffness and $50 \%$ of its stiffness, respectively. The peaks in Figure 1 
TABLE 1: A comparison between some terminologies used in both the BIS and the AIS.

\begin{tabular}{lll}
\hline Terminology & Definition in the BIS & Definition in the AIS for SHM \\
\hline Antigen & $\begin{array}{l}\text { A molecular pattern that can cause the reaction of the } \\
\text { immune system, which is usually harmful to the human } \\
\text { body. }\end{array}$ & $\begin{array}{l}\text { A data structure that consists of some related } \\
\text { information about a structural-health-condition. }\end{array}$ \\
\hline Antibody & $\begin{array}{l}\text { A portion of a receptor molecule that can bind with a } \\
\text { specific molecular pattern. }\end{array}$ & $\begin{array}{l}\text { A data structure that consists of some related } \\
\text { information about a structural-health-condition. This } \\
\text { structure is generated mathematically by the AIS. }\end{array}$ \\
\hline Affinity & $\begin{array}{l}\text { A measurement of the binding strength between an } \\
\text { antigen and an antibody. }\end{array}$ & $\begin{array}{l}\text { A criterion defined to scale the relationship between the } \\
\text { feature vector of an antigen and the one of an antibody. }\end{array}$ \\
\hline Memory cell & $\begin{array}{l}\text { An immune cell that contains information about } \\
\text { previously invading antigens. It stays in the immune } \\
\text { system and will initiate a secondary-immune-response } \\
\text { if it recognizes known antigens. }\end{array}$ & $\begin{array}{l}\text { A member in a database of AIS, which stores the data } \\
\text { structures of previous invading antigens. This database } \\
\text { is used by the AIS to recognize unknown } \\
\text { structural-health-conditions. }\end{array}$ \\
\hline $\begin{array}{l}\text { The process taken by the immune system to neutralize } \\
\text { invading antigens. }\end{array}$ & $\begin{array}{l}\text { The recognition process performed by the AIS to } \\
\text { determine unknown structural-health-conditions. }\end{array}$ \\
\hline
\end{tabular}

TABLE 2: Ten SPV sets used to train the AIS and test it.

\begin{tabular}{|c|c|c|c|c|c|c|c|c|}
\hline Set index & $m_{1}\left(10^{5} \mathrm{~kg}\right)$ & $m_{2}\left(10^{5} \mathrm{~kg}\right)$ & $m_{3}\left(10^{5} \mathrm{~kg}\right)$ & $k_{1}\left(10^{8} \mathrm{~kg} / \mathrm{s}^{2}\right)$ & $k_{2}\left(10^{8} \mathrm{~kg} / \mathrm{s}^{2}\right)$ & $k_{3}\left(10^{8} \mathrm{~kg} / \mathrm{s}^{2}\right)$ & $\alpha\left(\mathrm{s}^{-1}\right)$ & $\beta(s)$ \\
\hline 1 & 2.5 & 2.5 & 1.5 & 3.0 & 2.5 & 2.0 & 0.20 & 0.0015 \\
\hline 2 & 4.0 & 4.0 & 2.0 & 4.0 & 4.0 & 2.5 & 0.20 & 0.0015 \\
\hline 3 & 5.0 & 4.0 & 3.0 & 5.0 & 3.0 & 3.0 & 0.20 & 0.0015 \\
\hline 4 & 5.0 & 4.0 & 3.0 & 5.0 & 3.0 & 3.0 & 0.10 & 0.0020 \\
\hline 5 & 5.0 & 4.0 & 3.0 & 5.0 & 3.0 & 3.0 & 0.30 & 0.0010 \\
\hline 6 & 3.5 & 3.0 & 2.5 & 4.2 & 3.5 & 2.8 & 0.20 & 0.0015 \\
\hline 7 & 4.0 & 4.0 & 3.0 & 3.8 & 3.5 & 3.3 & 0.20 & 0.0015 \\
\hline 8 & 4.2 & 3.8 & 2.8 & 4.5 & 4.0 & 2.3 & 0.20 & 0.0015 \\
\hline 9 & 4.2 & 3.8 & 2.8 & 4.5 & 4.0 & 2.3 & 0.15 & 0.0018 \\
\hline 10 & 4.2 & 3.8 & 2.8 & 4.5 & 4.0 & 2.3 & 0.24 & 0.0021 \\
\hline
\end{tabular}

are the structural natural frequencies. It was observed that the natural frequencies decrease with the increasing damage severity.

The behavior of structural natural frequencies in different damage scenarios shows that the frequencies depend on the overall structure characteristics and different natural frequency has different sensitivity to damage in different locations. Namely, the natural frequencies exhibit a different changing pattern when the same damage severity happens in a different location. Based on this fact, this study intends to use the changing pattern in natural frequencies as the feature vector to monitor the structural-health-condition.

To extract the changing pattern of structural natural frequencies and minimize the influence of specific structure parameter values (SPVs), relative frequency changes (RFCs) were calculated by

$$
\Delta f_{\text {relative }}^{(i)}=\frac{f_{\text {healthy }}^{(i)}-f_{\text {damaged }}^{(i)}}{f_{\text {healthy }}^{(i)}},
$$

where the superscript $i\left(i=1,2, \ldots, N_{f}\right)$ is the index of the natural frequency and $N_{f}$ is the number of natural frequencies considered.
It was observed that the RFCs from different damage locations have similar values when the damage severity is small, which makes it difficult to discriminate different damage locations. To solve this issue, normalization was performed to the set of RFCs:

$$
\Delta f_{r, n}^{(i)}=\frac{\Delta f_{\text {relative }}^{(i)}}{\sqrt{\sum_{i=1}^{N_{f}}\left(\Delta f_{\text {relative }}^{(i)}\right)^{2}}},
$$

where $\Delta f_{r, n}^{(i)}$ is the normalized relative frequency change (NRFC).

The feature vector formed by the NRFCs represents the natural frequencies' changing pattern and is not sensitive to specific SPVs or damage severities. Feature vectors from two different SPV sets are shown in Figure 3.

Figures 3(a) and 3(b) were acquired from the simulations using the same structure model in Figure 2. The five cycles in each color represent five different damage severities (10\% to $50 \%$ at an interval of $10 \%)$ in the same damage location. The SPV sets used are set- 1 and set- 2 of Table 2 . To make results more obvious, feature vectors constructed by the NRFCs 


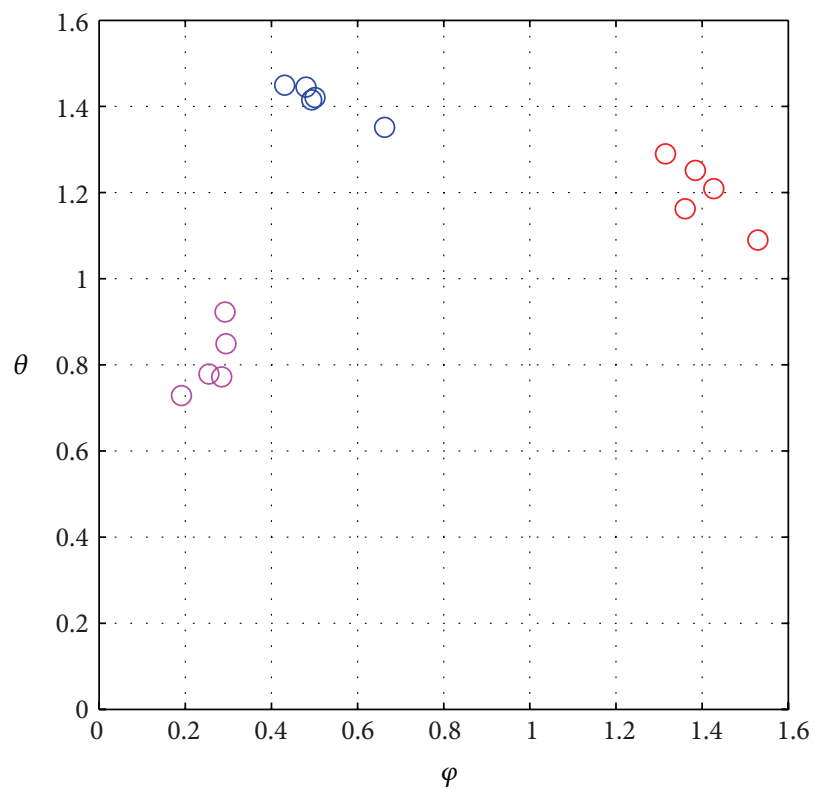

$k_{1}$ damage
$k_{2}$ damage
$k_{3}$ damage

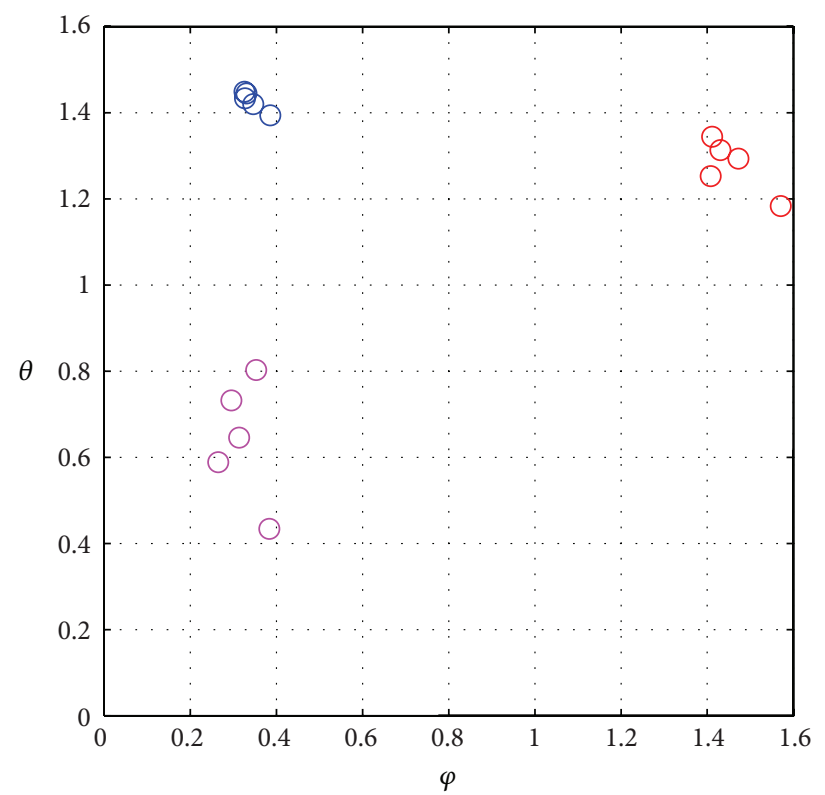

. $k_{1}$ damage

$k_{2}$ damage

$\bigcirc k_{3}$ damage

(a)

(b)

FIGURE 3: The feature space with points representing different structural-health-conditions. The feature vector was formed by the NRFCs and the simulation was performed using SPV (a) set-1 and (b) set-2.

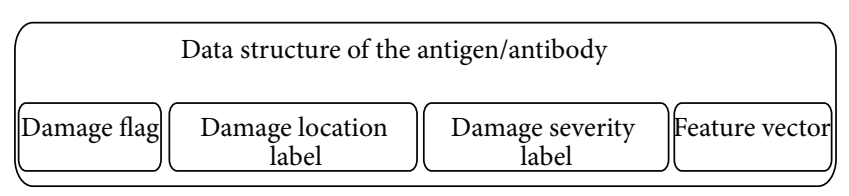

FIGURE 4: A schematic of the data structure of an antigen/antibody in the proposed AIS.

were converted from the Cartesian coordinate to a spherical coordinate using

$$
\begin{gathered}
r=\sqrt{x^{2}+y^{2}+z^{2}}, \\
\theta=\arccos \left(\frac{z}{r}\right), \\
\varphi=\arctan \left(\frac{y}{x}\right),
\end{gathered}
$$

where $x=\Delta f_{r, n}^{(1)}, y=\Delta f_{r, n}^{(2)}$, and $z=\Delta f_{r, n}^{(3)}$. Because of the normalization, the radii of feature vectors are always 1 (i.e., $r \equiv 1$ ). Therefore only $\varphi$ and $\theta$ were used to construct a feature vector, reducing the graphic dimensionality from three to two.

As shown in Figure 3, points representing the same damage location are clustered together in the feature space (i.e., the $\varphi-\theta$ plane), despite different SPVs and damage severities. In other words, the natural frequencies' changing pattern (represented by a set of NRFCs) is sensitive to the damage's location but is insensitive to either the SPV or the damage's severity. Therefore this feature vector formed by the NRFCs is used by the AIS in this paper to determine the location where damage occurs.

It was also observed in the simulation results that for the structural-health-conditions with the same damage location, the maximum RFC (mRFC) is roughly proportional to the damage's severity. Even with different SPVs, this proportional relationship is still similar. Therefore we employed the mRFC as an indicator of the damage's severity after the damage's location had been determined by the feature vector formed by the NRFCs.

\subsection{Construction of the Proposed Artificial Immune System} (AIS). In the proposed AIS, one antigen is defined as a data structure storing some information about one specific structural-damage-condition. This data structure consists of (1) a damage flag (is this structure damaged?), (2) a damagelocation-label (where is the damage?), (3) a damage-severitylabel (how serious is the damage?), and (4) a feature vector containing the characteristics of this damage (Figure 4). On the other hand, an antibody is defined to be the same data structure with an antigen, with the difference that antigens are generated from structural-damage-conditions while antibodies are generated mathematically by the AIS.

The learning process in the biological immune system (BIS) corresponds to the training process in the AIS, whose output (the memory cells set) is the vital component for 
the AIS' functionality. The training process is described as follows.

First, a number of training antigens were constructed from known structural-health-conditions. For each condition, its mRFC and NRFCs were calculated from the structural dynamic response and were stored in the training antigen's feature vector, while the damage's location and severity were recorded in the damage-location-label and the damage-severity-label, respectively.

Next, these training antigens were introduced to the untrained AIS one at a time, to stimulate a primary-immuneresponse. On the AIS' side, an initial-antibody-set was randomly generated to receive this stimulation.

With the stimulation from a training antigen, these initial antibodies carried out a clone process to generate more antibodies. The feature vectors of an antigen and an antibody are referred to in this paper as ag and ab, respectively. To mathematically quantify the intimacy between ag and ab, a variable named affinity was defined as

$$
\mathrm{aff}(\mathrm{ag}, \mathrm{ab})=\frac{1}{1+\operatorname{dis}(\mathrm{ag}, \mathrm{ab})},
$$

where function $\operatorname{dis}(\mathrm{ag}, \mathrm{ab})$ calculates the Euclidean distance between $\mathrm{ab}$ and ag in the feature space.

Based on the BIS' principles, a higher affinity value between ab and ag means this antibody will have more clones. Thus the clone number $(\mathrm{CN})$ of this antibody in the close process was calculated using

$$
\mathrm{CN}=\operatorname{round}(\mathrm{CR} \cdot \mathrm{aff}(\mathrm{ag}, \mathrm{ab})),
$$

where CR is a prespecified clone rate and function round( ) returns its argument's nearest integer.

After the clone, the antibody set underwent a mutation process to increase its diversity and possibly generate antibodies having higher affinity values with the training antigen. Because the antibodies having higher affinity values with the antigen experience smaller mutation extent in the BIS, the mutation process in the AIS was defined by

$$
\mathrm{ab}_{m}=\mathrm{ab}+\mathrm{MR} \cdot \delta
$$

where $a b_{m}$ is the feature vector of a mutated antibody, $\delta$ is a standard normal random variable having the same dimensions with the feature vector, and MR is the mutation rate, which was defined as

$$
\mathrm{MR}=1-\mathrm{aff}(\mathrm{ab}, \mathrm{ag}) .
$$

Following the clone and mutation processes, the affinity values between all antibodies and the training antigen were calculated using (4). The antibodies with an affinity value higher than a prespecified candidate selection threshold (CST) were sifted as the candidate-memory-cells and were considered to have the same damage location with the training antigen.

Then, these candidate-memory-cells were introduced into the existing memory cell set one-by-one. For each candidate, if there was no memory cell having the same damage location with it, it was injected into the memory cell set directly. Otherwise, if there were memory cells having the same damage location with this candidate, the affinity values between these memory cells and this candidate were calculated. If the maximum affinity value was lower than a prespecified candidate injection threshold (CIT), this candidate was injected into the memory cell set. Otherwise, this candidate was discarded.

The AIS' reaction process to a training antigen ended here and this process is schematically illustrated in Figure 5 . The immune system's training process finished when all training antigens had gone through this process. At this time, the memory cell set was named as the mature memory cell set.

From the discussion in Section 2.1, the changing pattern of the structural natural frequencies represented by the set of NRFCs is sensitive to the damage's location but insensitive to the damage's severity. Therefore another part involving the mRFC was added to the AIS to estimate the damage's severity.

To build the algorithm for the damage-severityestimation, one linear regression was performed between the mRFCs of training antigens in the same damage location and their damage severities. Each possible damage location has one regression, and these regressions were set to have zero intercept. The slopes of these regressions were recorded in the AIS as the estimation coefficients for each corresponding damage location.

After the steps in Figure 5, the AIS is ready to determine unknown structural-health-conditions. This determination is a 3-stage procedure (Figure 6): the damage-existencedetection, the damage-location-determination, and the damage-severity-estimation.

Before applying the trained AIS, one unknown structuralhealth-condition needs to be converted into a compatible data structure (i.e., antigen). The dynamic response of the structure in an unknown structural-health-condition was acquired and analyzed using the aforementioned procedures to form the feature vector and furthermore to form the unknown antigen. The word "unknown" here means that the trained AIS knows nothing about the antigen except for its feature vector. This unknown antigen was introduced into the trained AIS.

The trained AIS first performed the damage-existencedetection: if the mRFC of this antigen was smaller than a prespecified damage threshold (DT), this antigen was classified as "self" and labeled as "healthy." Otherwise, the trained AIS labeled this condition as "damaged" and moved on to the damage-location-determination.

In the damage-location-determination, the affinity values between each memory cell and this antigen were calculated. If the highest affinity was higher than the authentic threshold (AT), this antigen was classified to have the same damage location with its nearest memory cell, and this result was considered to be "authentic," which means the immune system was pretty "confident." On the other hand, if the highest affinity was lower than the AT, this antigen was still classified to have the same damage location with its nearest memory cell, but this result was considered to be "unauthentic," which means the immune system was not "sure" about this result. The two thresholds (DT and AT) need 


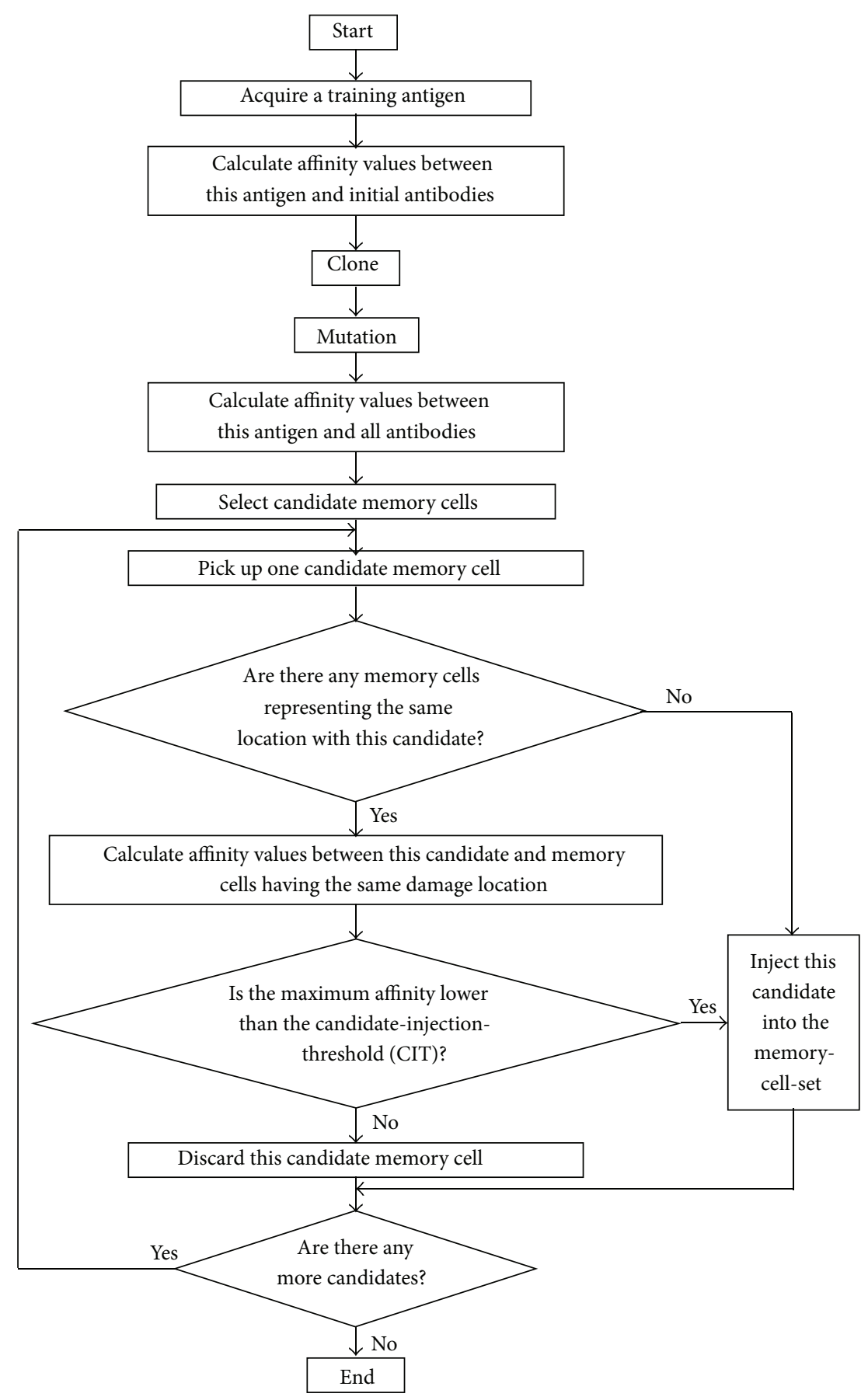

FIGURE 5: A schematic illustration of the AIS' reaction process to a training antigen.

to be selected empirically. The influence of different threshold values on the AIS' effectiveness and efficiency requires further investigation and will be covered in following researches.

In the last stage, estimation about the antigen's damage severity was made using its $\mathrm{mRFC}$ and the estimation coefficient corresponding to its damage location. Finally, the trained AIS outputted the estimated healthy condition, whether or not the structure is damaged, if yes, where the damage location is and what the damage severity is.

\section{Performance Evaluations}

3.1. Simulation Setup and Parameter Values Selection. The performance of the proposed AIS for the SHM was evaluated by a linear 3-floor structure model (Figure 2), which was simplified to a linear 3-degree-of-freedom (3DOF) mathematic model with the governing equation

$$
M \ddot{X}+C \dot{X}+K X=F
$$




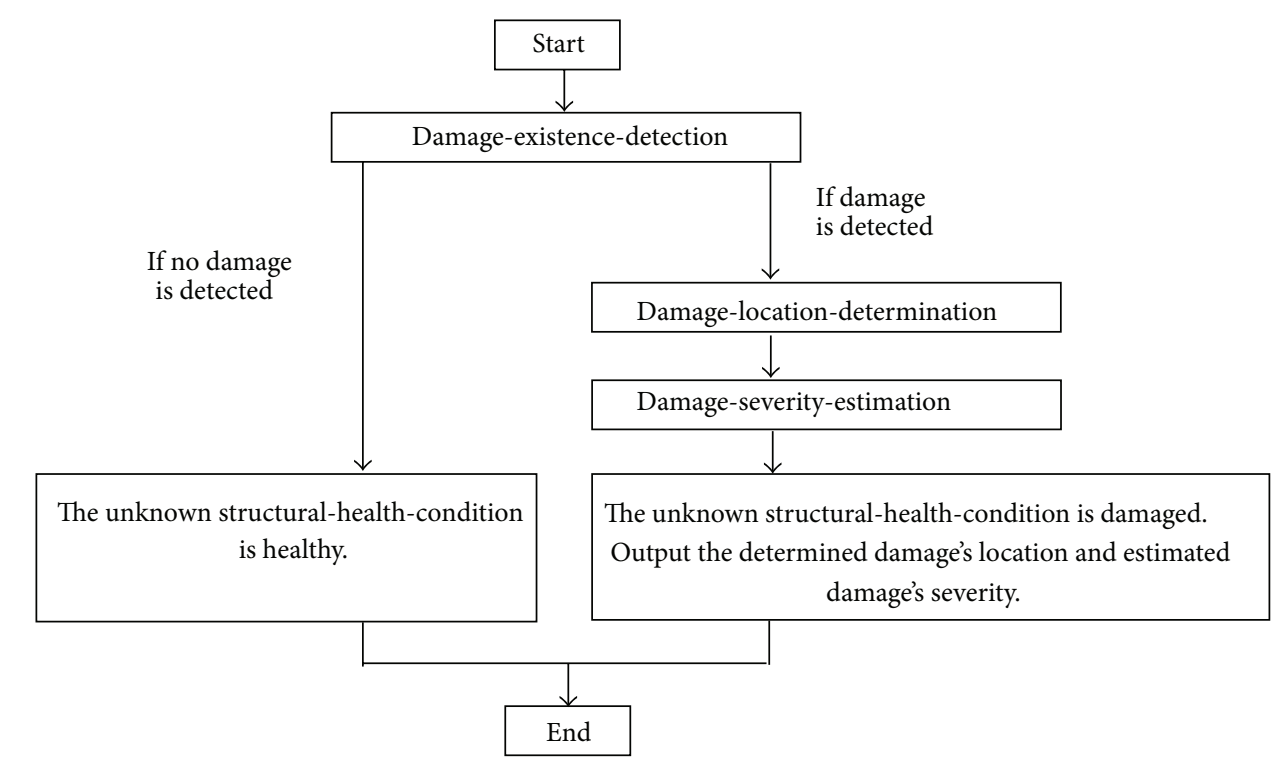

FIGURE 6: The flow chart of the 3-stage procedure to determine an unknown structural-health-condition.

where $X=\left[\begin{array}{lll}x_{1} & x_{2} & x_{3}\end{array}\right]^{\mathrm{T}}$ is the displacement vector, $F=$ $\left[\begin{array}{lll}f_{1} & f_{2} & f_{3}\end{array}\right]^{\mathrm{T}}$ is the external force vector, and

$$
M=\left[\begin{array}{ccc}
m_{1} & 0 & 0 \\
0 & m_{2} & 0 \\
0 & 0 & m_{3}
\end{array}\right], \quad K=\left[\begin{array}{ccc}
k_{1}+k_{2} & -k_{2} & 0 \\
-k_{2} & k_{2}+k_{3} & -k_{3} \\
0 & -k_{3} & k_{3}
\end{array}\right]
$$

are the system's mass matrix and stiffness matrix, respectively. Rayleigh damping was assumed; that is,

$$
C=\alpha M+\beta K
$$

where $\alpha$ and $\beta$ are prespecified parameters. The superscript T denotes transpose.

10 different structure-parameter-value (SPV) sets were created (Table 2) based on [13-15]. The first 5 sets were used to train the AIS and then all the 10 sets were used to test the AIS’ performance.

Simulations were performed by MATLAB using a selfprogrammed 4th-order Runge-Kutta algorithm. Nonzero initial displacement condition was used to excite the structure into free vibration. The displacements of all three floors were recorded for $30 \mathrm{sec}$ at $1000 \mathrm{~Hz}$. Then the fast Fourier transform (FFT) was employed to transfer the displacement data of the first floor from the time domain to the frequency domain, from where structural natural frequencies (peaks in the graph) were extracted. Different structural damages were simulated by reducing different stiffness values (variables of $K$ in (8)) by different extents. The single damage scenario was assumed, meaning that only one of the three stiffness variables $\left(k_{1}, k_{2}\right.$, and $\left.k_{3}\right)$ was reduced in each structural damage condition.

3.2. Training of the Artificial Immune System (AIS). To train the AIS, 5 healthy and 75 damaged conditions were used.
They represent different structural-health-conditions in the first 5 structural-parameter-value (SPV) sets of Table 2. Each SPV set has 1 healthy condition and 15 damaged conditions (damage location (1st, 2nd, and 3rd floor) and damage severities (stiffness loss of $10 \%$ to $50 \%$ at an interval of $10 \%$ )).

Dynamic responses of the structure in different SPV sets and structural-health-conditions were simulated using the previous described setup. Structural natural frequencies were extracted and used to calculate the mRFCs using (1) and NRFCs suing (2). The normalization process makes the Euclidean norm of the set of NRFCs always equal to unity, meaning the points representing sets of NRFCs are all on the surface of a sphere (or hypersphere). In our case $\left(N_{f}=\right.$ 3 ), a coordinate conversion from the Cartesian coordinate to a spherical coordinate using (3) was performed to make results more obvious. Because $r \equiv 1$, only $\theta$ and $\varphi$ were used to represent the point and to form the feature vectors. The procedure to form feature vectors is illustrated in Figure 7.

In Figure 7, process 1 extracts the structural natural frequencies from the dynamic response; process 2 calculates the RFCs using (1); process 3 normalizes RFCs using (2); process 4 shows the set of NRFCs in an Cartesian coordinate; process 5 converts the Cartesian coordinates to spherical coordinates and discards the radius variable; and finally process 6 shows the set of converted coordinates in the $\varphi-\theta$ plane. The set of coordinates after process 6 is the feature vector of this structural-health-condition.

Using procedures described in Figure 7, feature vectors from 80 training conditions were acquired. Among them, the 5 feature vectors from healthy conditions were used as the baselines to calculate the RFCs. Points representing the rest 75 damaged conditions are plotted in Figure 8.

For the training stage, the prespecified variables were selected as follows. The number of initial antibodies is 10000 , the clone rate $\mathrm{CN}=10$, the candidate selection threshold CST $=0.9$, and the candidate injection threshold CIT $=0.98$. 

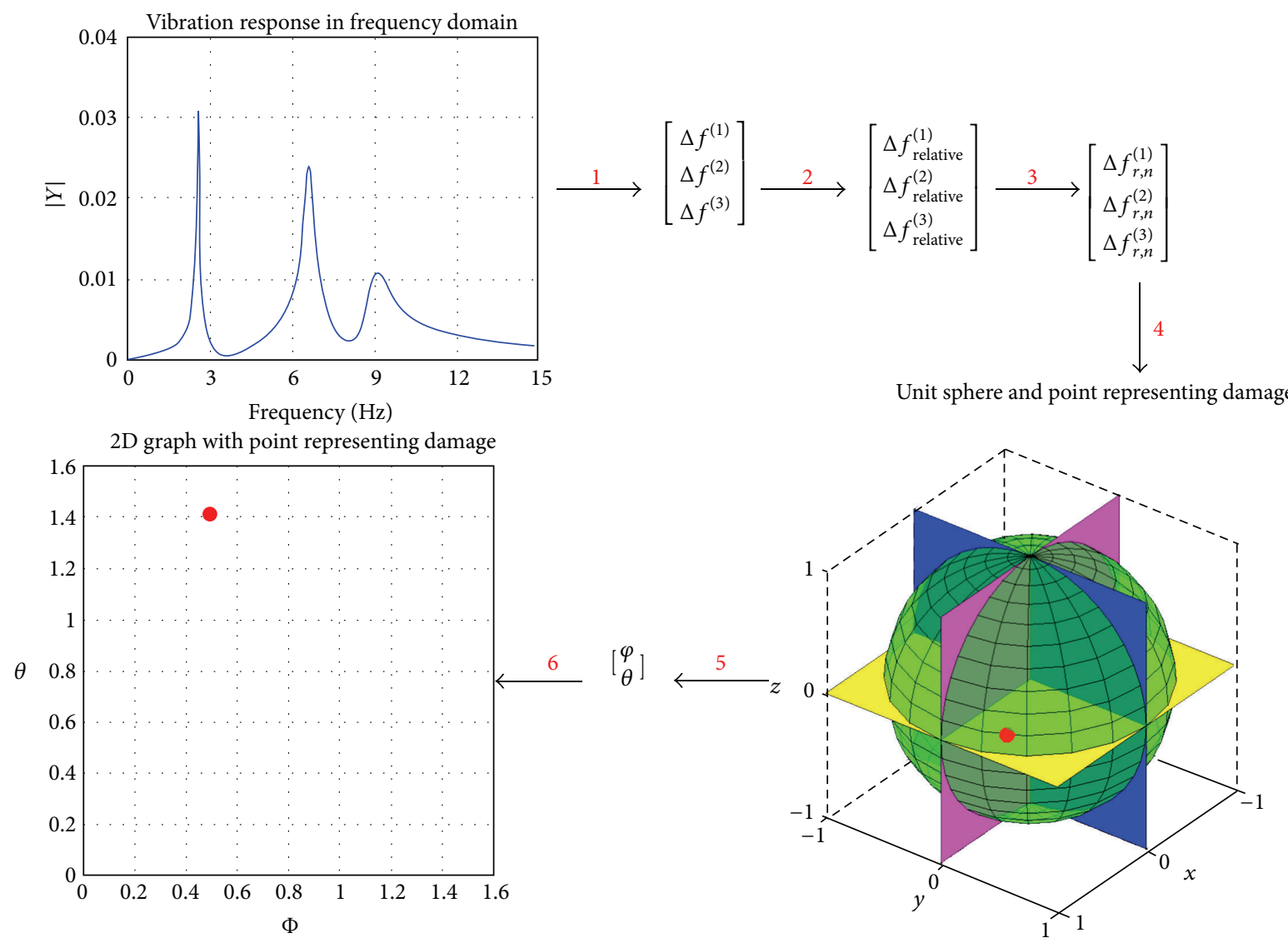

FIGURE 7: Schematic of the procedures to form a feature vector using a simulation result.

The AIS was trained using the 75 known damaged conditions and the procedures described earlier. It should be noticed that the values of some feature vectors might exceed the range of $[0, \pi / 2]$ after the mutation process. To make sure all feature vectors are inside this range, if a mutated antibody had feature vector values bigger than $\pi / 2$, these values were reduced to be $\pi / 2$; if it had feature vector values smaller than 0 , these values were increased to be 0 . Figure 9 shows the mature memory cells after the AIS' training process.

To develop the damage-severity-estimator, the severity estimation coefficients were acquired from the linear regressions between the damage severities and the mRFCs for each damage location (Figure 10). The slope of the regression function was taken as the estimation coefficient for this damage location. From Figure 10, the estimation coefficients were 2.8087 for the 1st floor damage, 3.1759 for the 2nd floor damage, and 2.6456 for the 3rd floor damage.

3.3. Validation of the Trained Artificial Immune System (AIS). To evaluate the performance of the trained AIS, 100 damage severities were randomly generated inside the range of 0 $50 \%$ for each damage location, and a total of 3000 damaged conditions were used (300 for each structural parameter set (SPV) in Table 2). Healthy conditions of the 10 SPV sets were also simulated and used as the baseline data to calculate the feature vectors of damaged conditions.
Simulation results from the 3000 damaged conditions were used to form the unknown antigens using the procedure described in Figure 7, which were then introduced into the trained AIS. The AIS estimated the unknown antigens using procedures described in Section 2.3. The estimation included the information of whether or not this condition is healthy, if not, where the damage is and how severe the damage is. In this estimation process, the damage threshold (DT) used for the damage-existence-detection and the authentic threshold (AT) were set to be $1 \%$ and 0.9 , respectively.

3.4. Performance Analysis. Among the 3000 testing conditions, the AIS succeeded in detecting the damage existence in 2890 cases and failed in the other 110 cases. The failures are because the mRFCs in these cases were smaller than the damage threshold (DT). Real damage severities in these "missed" cases were smaller than $5 \%$, suggesting that the natural frequencies are not sensitive enough to catch minor damages.

In the 2890 success cases, 2760 cases were "authentic" and 130 cases were "unauthentic." The difference between the "authentic" and "unauthentic" results is whether or not the AIS is "confident" with the result. If the highest affinity value between a feature vector and memory cells was higher than the AT, the immune system was "confident" with its estimation. Otherwise, the immune system was not "confident." 


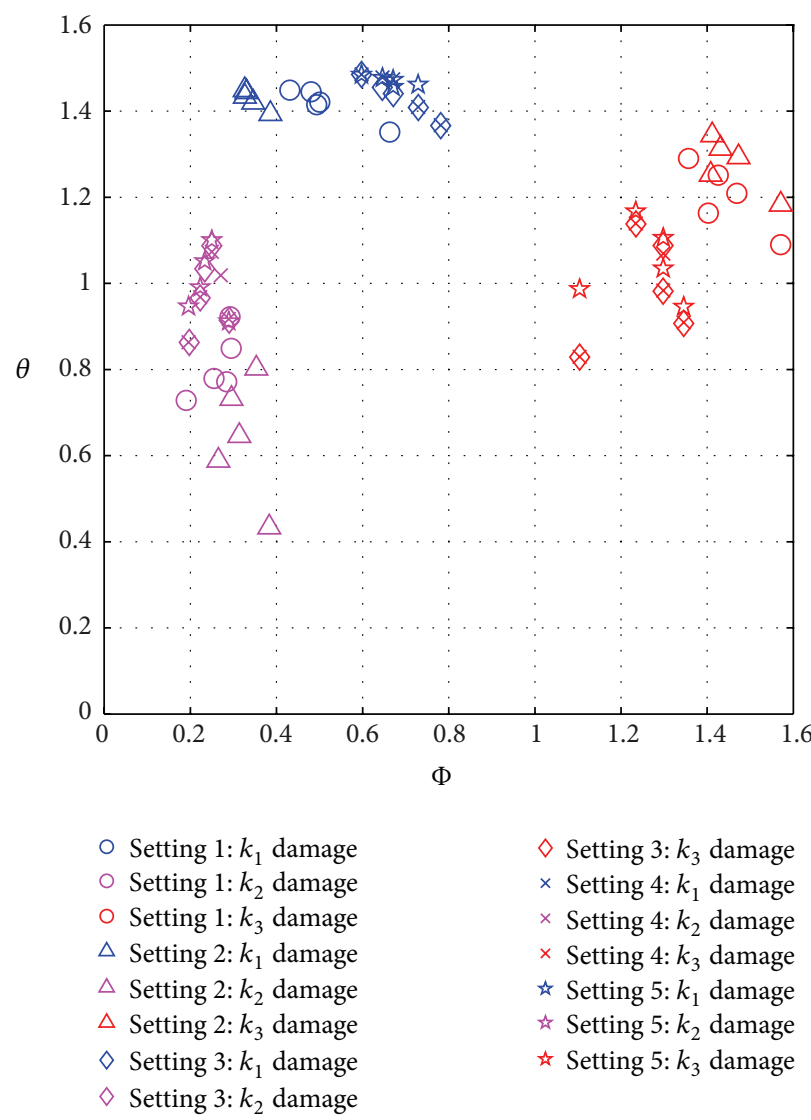

FIGURE 8: A graph with points representing feature vectors of the 75 damaged conditions for the training of the AIS.

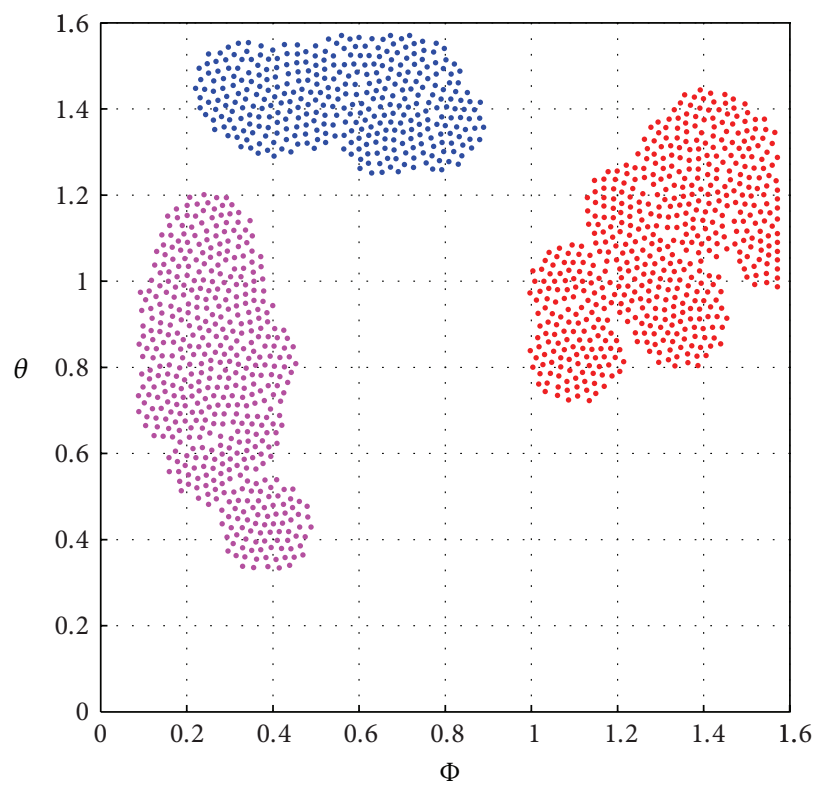

- $k_{1}$ damage memory cell

- $k_{2}$ damage memory cell

- $k_{3}$ damage memory cell

FIGURE 9: A graph with points representing the outcome of the AIS’ training: the mature memory cells. 

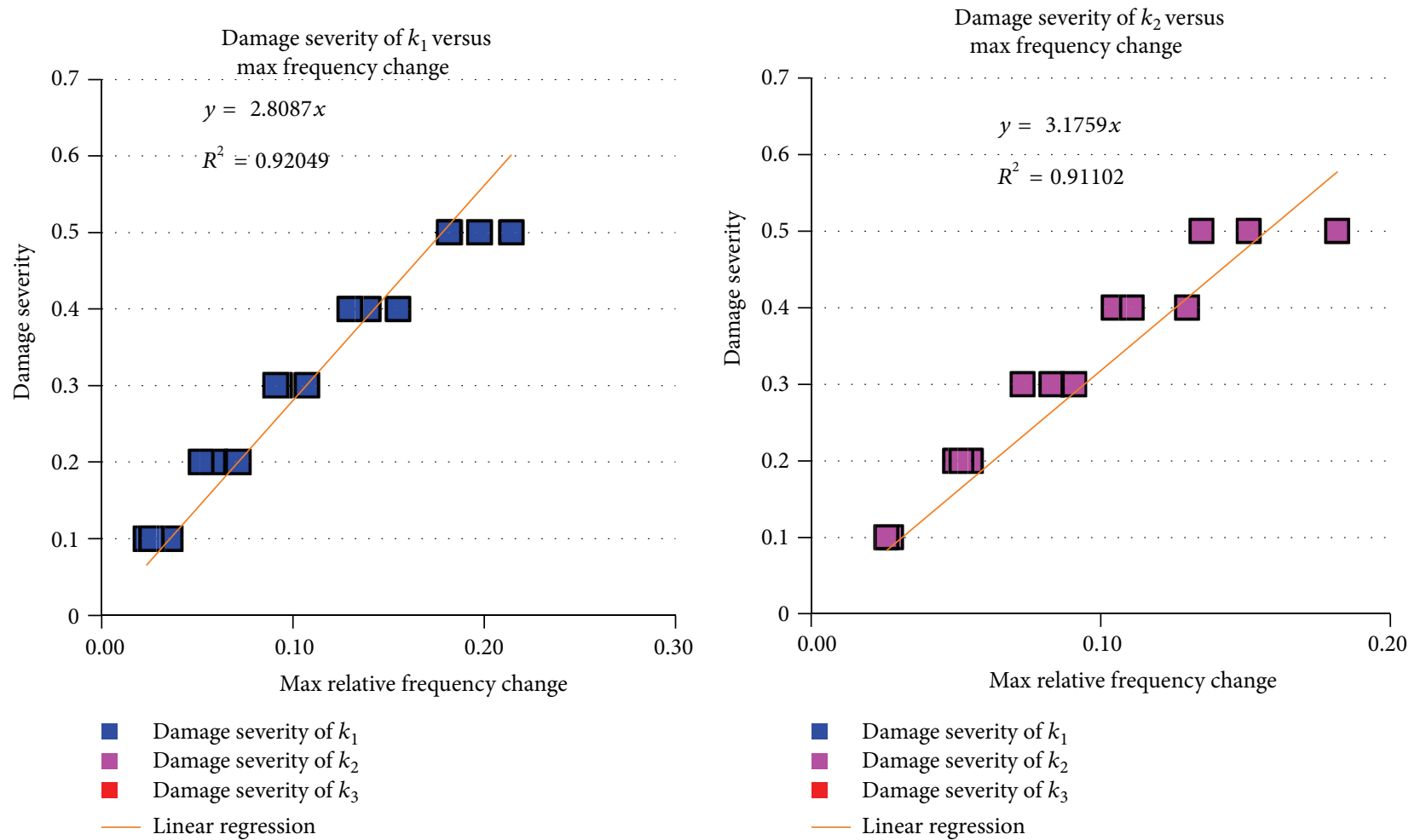

(a)

(b)

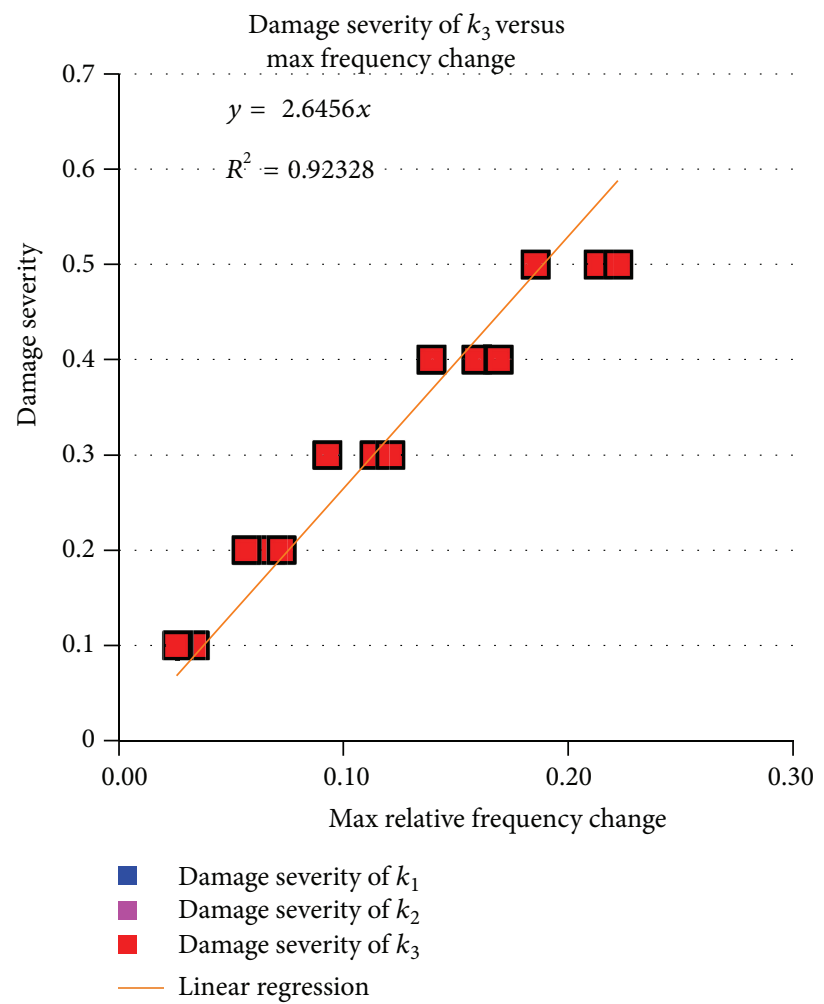

(c)

FIGURE 10: Regressions between the mRFCs and their corresponding damage severities for each damage location: (a) for the 1st floor damage, (b) for the 2nd floor damage, and (c) for the 3rd floor damage. 


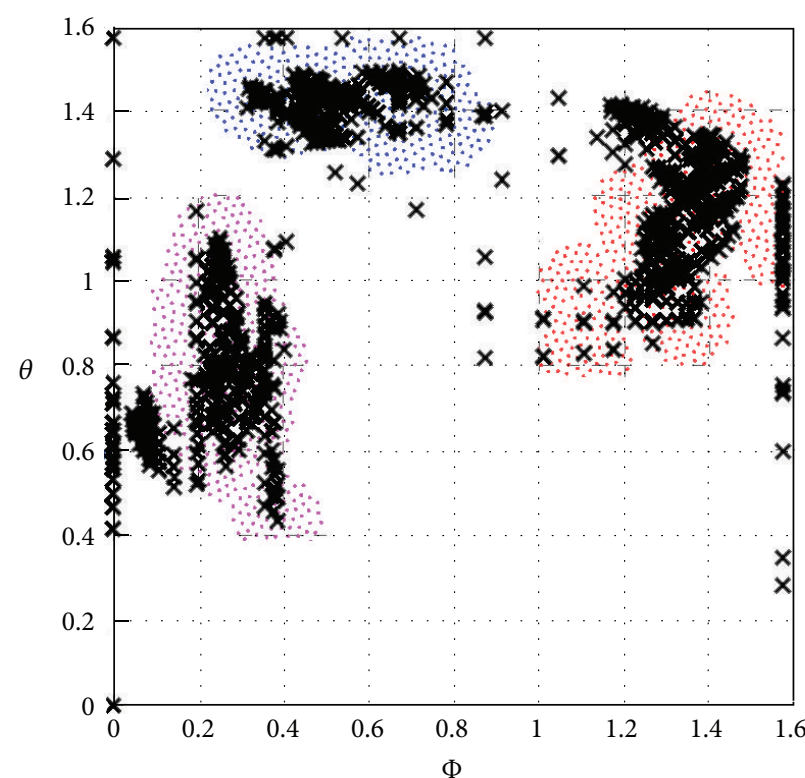

- $k_{1}$ damage memory cell

- $k_{2}$ damage memory cell

- $k_{3}$ damage memory cell

$\times$ Unknown damage situation

(a)

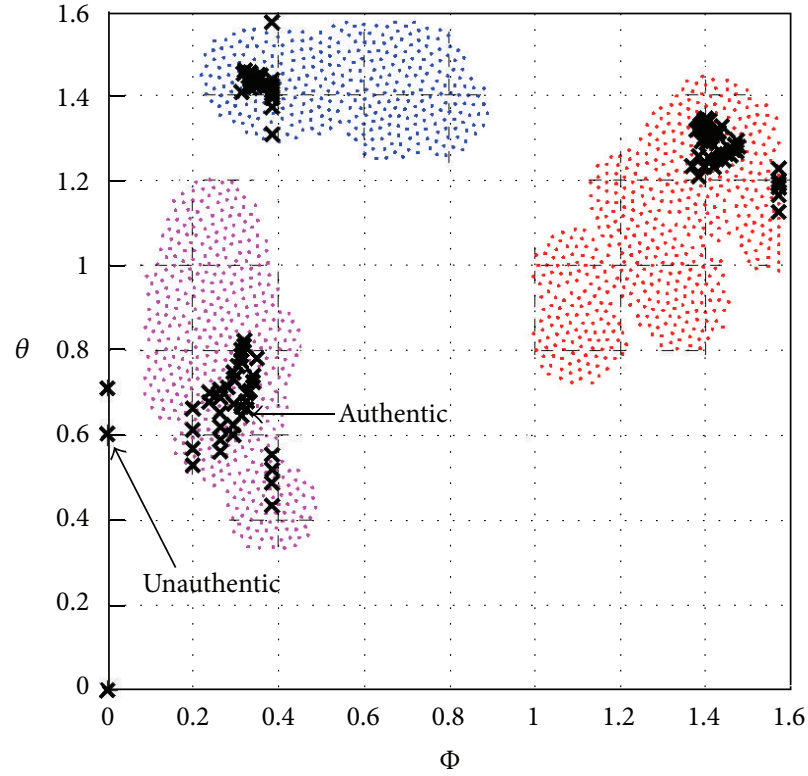

- $k_{1}$ damage memory cell

- $k_{2}$ damage memory cell

- $k_{3}$ damage memory cell

$\times$ Unknown damage situation

(b)

Figure 11: Mature memory cells and unknown antigens from (a) SPV set-1 to set-10 and (b) set-2.

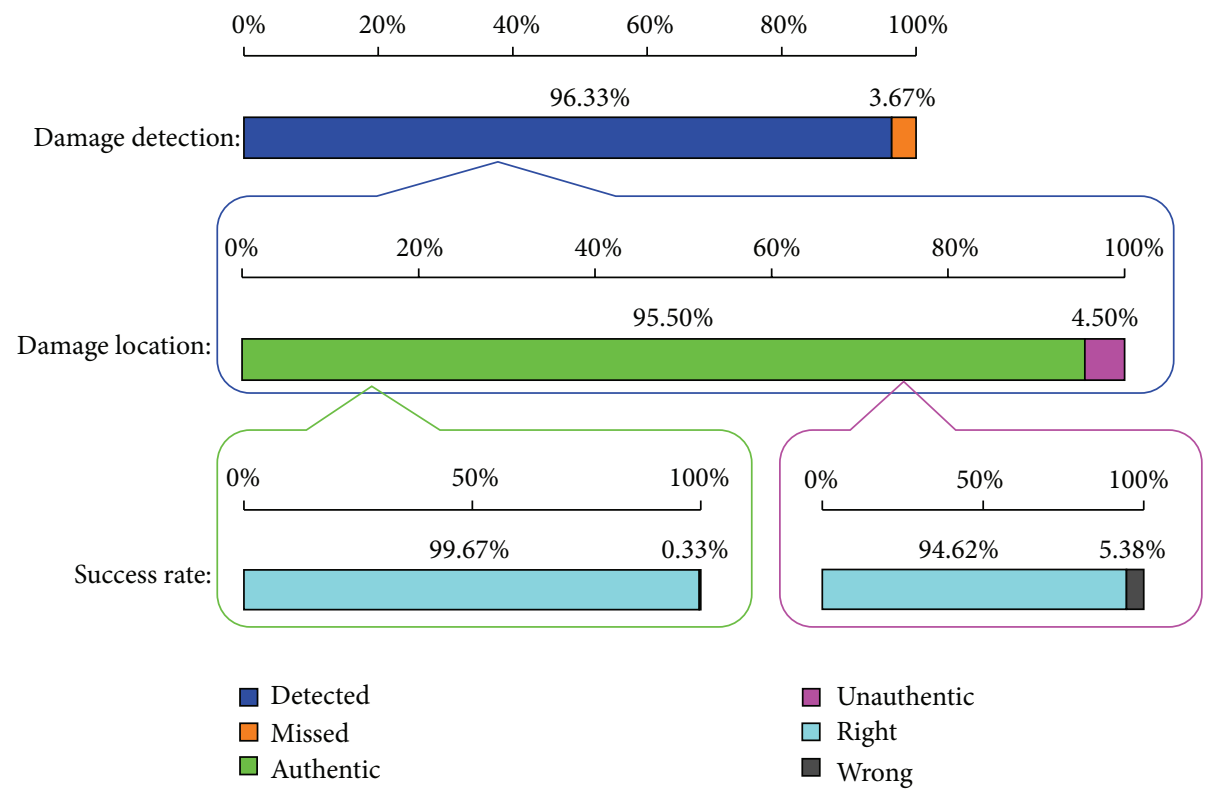

FIGURE 12: Performance of the proposed AIS in the damage-existence-detection and the damage-location-determination tasks.

All unknown antigens are presented in Figure 11(a) and an illustration of "authentic" results and "unauthentic" results is shown in Figure 11(b).

Among the 2760 "authentic" cases, the AIS correctly pointed out to the damage locations in 2751 cases (success rate is $99.67 \%)$. In the 130 "unauthentic" cases, the AIS correctly pointed out to the damage locations in 123 cases (success rate is 94.62\%). This result is shown in Figure 12. The overall success rate of this AIS in the damage-existencedetection and the damage-location-determination is $95.80 \%$. In failed cases, real damage severities were less than $6 \%$, which implies that the inaccuracy of the AIS is possibly due 


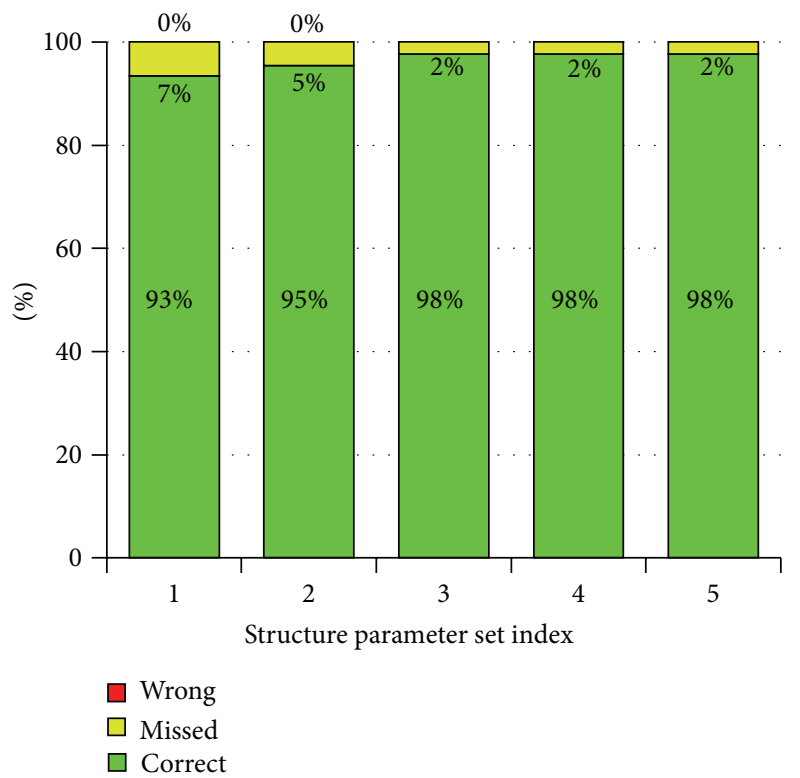

(a)

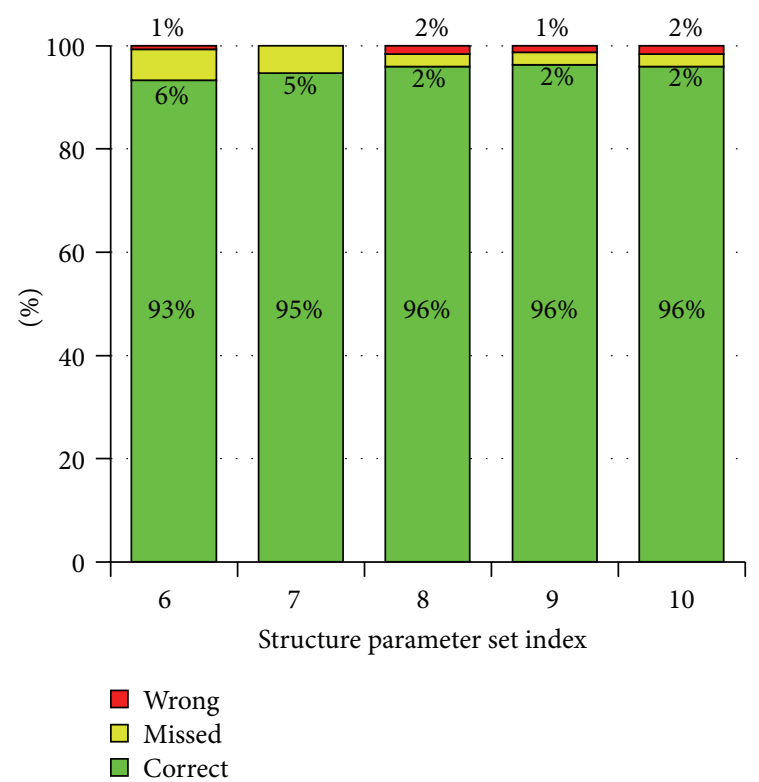

(b)

FIGURE 13: Performance of the proposed AIS in the damage-existence-detection and the damage-location-determination tasks with (a) SPV set $1-5$ and (b) SPV set $6-10$.

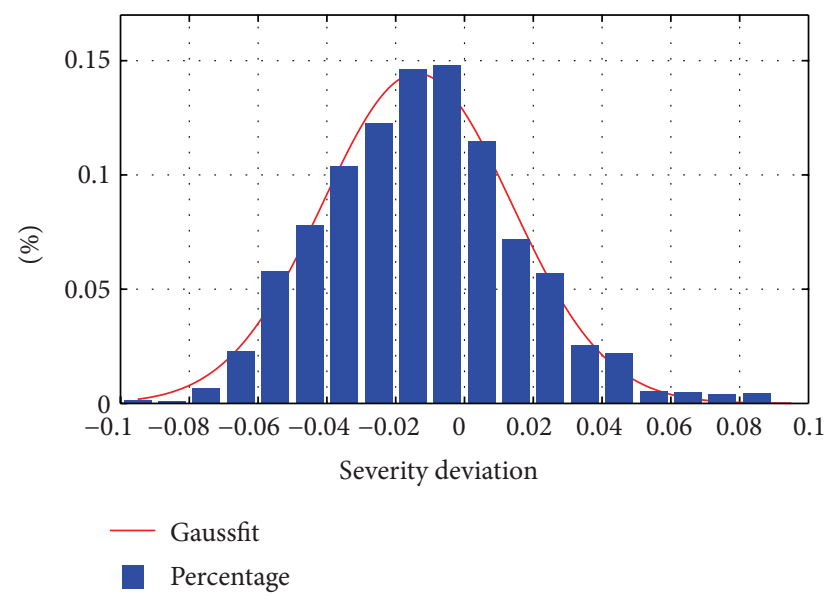

FIGURE 14: Distribution of the SDs of the AIS' severity estimations in "correct" cases and the "best-fit" Gauss curve.

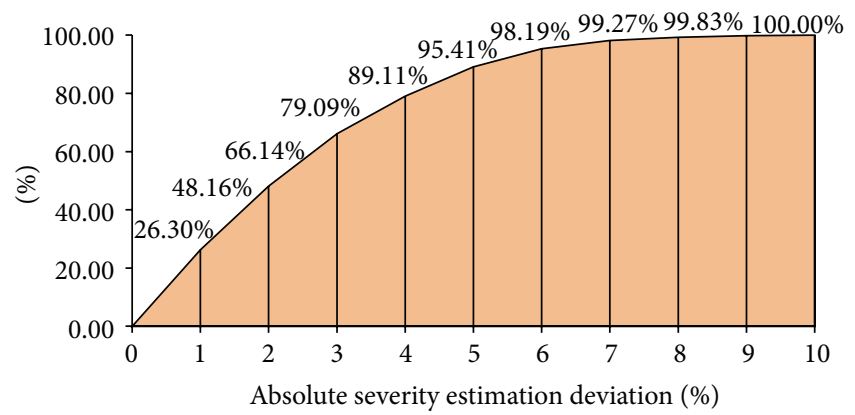

FIGURE 15: The accumulative percentage of the absolute SD of the AIS’ severity estimations. to the insensitivity of the natural frequencies to small damage severities.

Performances of the trained AIS to damaged conditions from different SPV sets are shown in Figure 13. No significant difference was observed between performances in different SPV sets. In set- 1 and set-6, the "unrecognized" rates were high because natural frequencies in these two sets were less sensitive to damages on the 2nd floor compared with other SPV sets. Therefore the mRFCs of more conditions were smaller than the DT and more conditions were labeled as "healthy" by the trained AIS, resulting in a higher "missed" rate.

It can be seen that all "wrong" results happen in Figure 13(b), which presents results from SPV sets that did not appear in the training stage. Also, the authentic rate of detected cases in the last 5 sets $(93.01 \%)$ was lower than the authentic rate in the first 5 sets $(97.99 \%)$, which implies that the SPV difference between the tested structures and structures used for training does have a negative effect on the AIS' performance. But as indicated by Figure 13, this negative effect is not significant.

To quantify this trained AIS' performance in the damageseverity-estimation, the severity deviation (SD) was defined as the difference between the estimated and real damage severity. The distribution of the SDs of "correct" cases in damage existence and location determination is shown in Figure 14, where the red line is a "best-fit" Gauss curve to the data points with

$$
P=0.1444 e^{-((x+0.0138) / 0.0388)^{2}} .
$$

If the absolute value of the SD is considered, $89.11 \%$ results deviate from the real severity for less than $5 \%$ (Figure 15). 


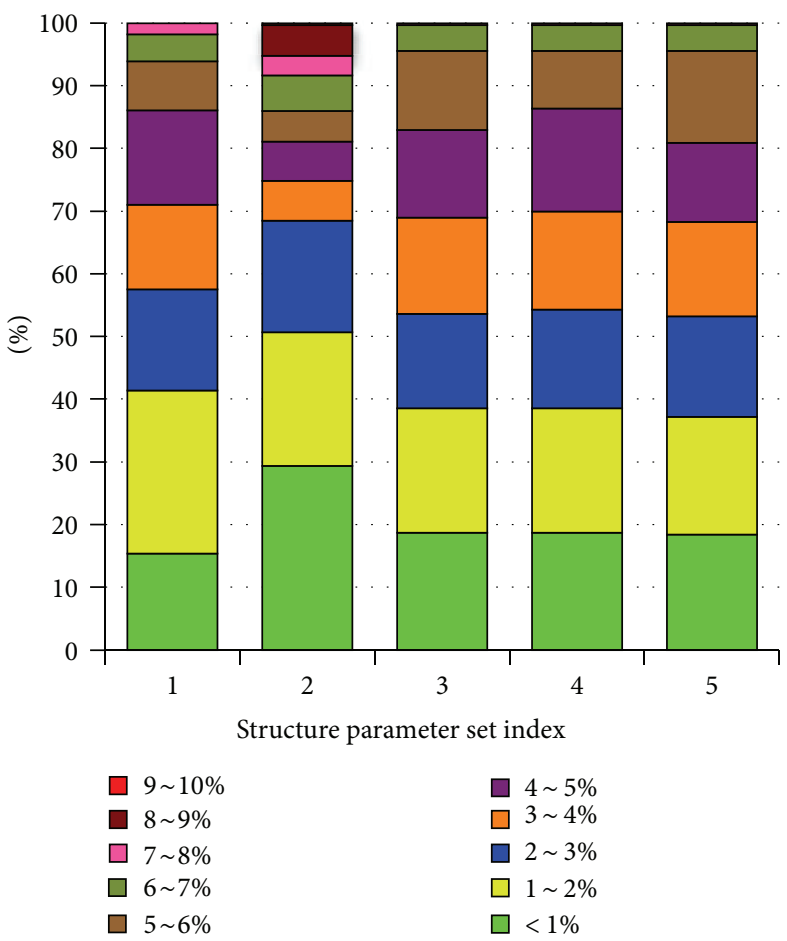

(a)

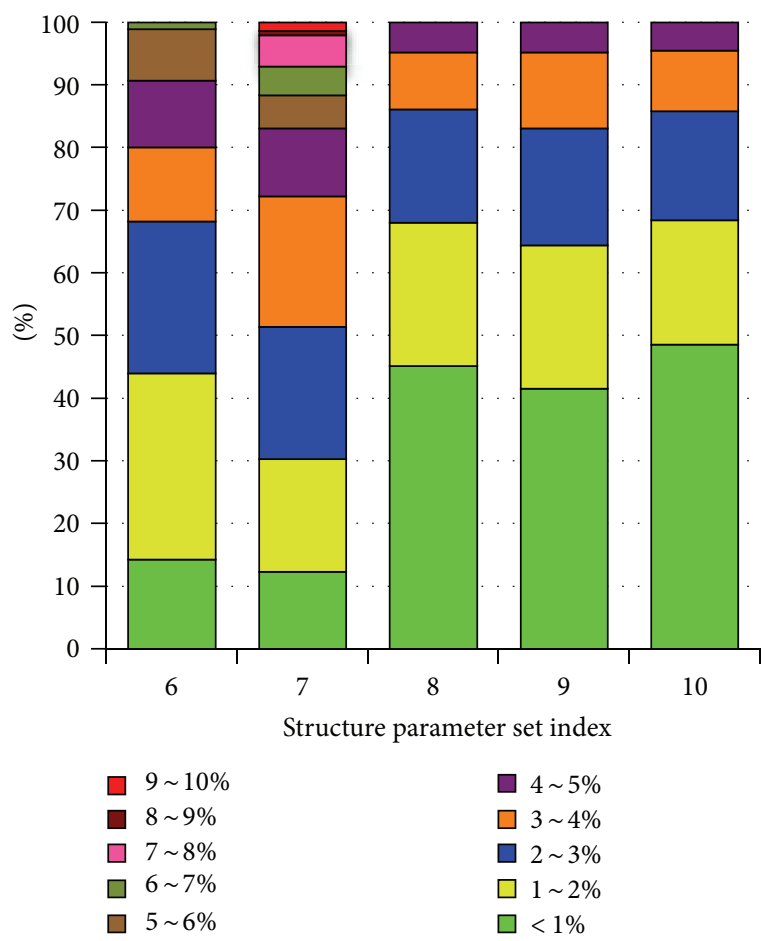

(b)

FIGURE 16: Severity deviation distribution of the artificial immune system's performances in (a) parameter set 1-5 and (b) parameter set 6-10.

Similar to the situation in the damage-existencedetection and the damage-location-determination, in the damage-severity-estimation, there was no significant difference between the AIS' performances in different SPV sets (Figure 16). The distribution of the SD shows that this AIS is capable of providing relatively accurate damage-severity-estimation if the damage location is known.

The trained AIS exhibited similar performance on SPV set- 3 , set- 4 , and 5 , and set- 8 , set- 9 , and set- 10 . This is believed to be a result of set- 3 , set- 4 , and set- $5(8,9$, and 10) having the same mass and stiffness values, but different damping ratios. This suggests that the damping ratio has a less significant influence on the AIS' performance than the mass and stiffness.

The above results showed that, once trained, the proposed AIS can be applied to the SHM on structures with different SPVs with satisfying performance. However, different SPVs do have different effect on the AIS' performance. If the SPV set is included in the training stage, the trained AIS is more likely to make a "confident" ("authentic") estimation and its success rate is relatively higher. Otherwise, if the SPV set does not appear in the training stage, the trained AIS is less "confident" and its success rate is relatively lower.

\section{Conclusions}

This paper proposes a three-step AIS-based approach for the SHM of civil structures. The two major advantages of the proposed method over other existing similar approaches are (1) relatively wide applicability. The proposed AIS is less sensitive to the structural-parameter-values (SPVs) than other existing AIS schemes. Once the proposed AIS is trained, it could be applied to several similar structures and the differences between these structures won't significantly deteriorate the trained AIS' performance. This makes the proposed AIS more economic and practically feasible than the schemes requiring unique training for each individual structure. (2) Low demand for the training conditions. The results showed that the proposed AIS could reach a relatively high accuracy with limited training antigens.

There are three stages in the proposed AIS: the damageexistence-detection, the damage-location-determination, and the damage-severity-estimation, whose relationship is shown in Figure 6. The proposed AIS uses the structuralnatural-frequencies as the damage sensitive variables. The mRFC and the NRFCs are used by the AIS' feature vector. The mRFC is used for the damage-existence-detection and the damage-severity-estimation, while the NRFCs are used for the damage-location-determination. The AIS' training process to get a mature memory cell set using known structural-health-conditions is minutely discussed.

A linear three-floor structure model was employed to demonstrate and validate the proposed AIS. The AIS was trained using 75 known structural-health-conditions in 5 different SPV sets and was then applied to 3000 unknown structural-health-conditions from 10 different SPV sets, 5 of the 10 SPV sets used for the validation did not appear in the training process. Results showed that the proposed AIS is capable of detecting the damage's existence, determining the 
damage's location, and estimating the damage's severity with a relatively high accuracy. It also showed that the proposed AIS only needs a small number of representative structuralhealth-conditions for its training to achieve a satisfying performance.

\section{Future Research Directions}

This paper only presents the idea of the proposed AIS and some primary simulation results, which were obtained from a linear three-degree-of-freedom (3DOF) structure model. The involved conditions were assumed to be ideal for simplicity and thus many practical issues were not included, such as the environment noise, the parameters' selection, the number of degree-of-freedom, the structural-naturalfrequency extraction, and the nonlinearity. These issues need further investigation and some of them were studied in the following research, that is, applications of the proposed AIS to structures with different number of degree-of-freedom and to the benchmark structure proposed by the International Association for Structural Control-American Society of Civil Engineers (IASC-ASCE) Structural Health Monitoring Task Group. Some results of the following research can be found in [16].

\section{Conflict of Interests}

Jiachen Zhang and Zhikun Hou declare that there is no conflict of interests regarding the publication of this paper.

\section{References}

[1] C. R. Farrar and K. Worden, "An introduction to structural health monitoring," Philosophical Transactions of the Royal Society A, vol. 365, no. 1851, pp. 303-315, 2007.

[2] K. Worden, C. R. Farrar, G. Manson, and G. Park, "The fundamental axioms of structural health monitoring," Proceedings of the Royal Society A Mathematical, Physical and Engineering Sciences, vol. 463, no. 2082, pp. 1639-1664, 2007.

[3] "Structural health monitoring: research and applications", in Proceedings of the Asia-Pacific Workshop on Structural Health Monitoring, W. K. Chiu and S. C. Galea, Eds., p. 28, Melbourne, Australia, 2013.

[4] D. Dasgupta, S. Yu, and F. Nino, "Recent advances in artificial immune systems: models and applications," Applied Soft Computing Journal, vol. 11, no. 2, pp. 1574-1587, 2011.

[5] S. Forrest, L. Allen, A. S. Perelson, and R. Cherukuri, "Selfnonself discrimination in a computer," in Proceedings of the IEEE Symposium on Research in Security and Privacy, pp. 202212, May 1994.

[6] P. E. Seiden and F. Celada, "A model for simulating cognate recognition and response in the immune system," Journal of Theoretical Biology, vol. 158, no. 3, pp. 329-357, 1992.

[7] L. N. de Castro and F. J. Von Zuben, "Learning and optimization using the clonal selection principle," IEEE Transactions on Evolutionary Computation, vol. 6, no. 3, pp. 239-251, 2002.

[8] B. Chen and C. Zang, "Artificial immune pattern recognition for structure damage classification," Computers and Structures, vol. 87, no. 21-22, pp. 1394-1407, 2009.
[9] B. Chen and C. Zang, "A hybrid immune model for unsupervised structural damage pattern recognition," Expert Systems with Applications, vol. 38, no. 3, pp. 1650-1658, 2011.

[10] W. Xiao, Structural health monitoring and fault diagnosis based on artificial immune system [M.S. thesis], Worcester Polytechnic Institute, Worcester, Mass, USA, 2012.

[11] Immune System, "The gale encyclopedia of science. Detroit," Gale, vol. 3, pp. 2246-2254, 2008.

[12] S. W. Doebling, C. R. Farrar, M. B. Prime, and D. W. Shevitz, "Damage identification and health monitoring of structural and mechanical systems from changes in their vibration characteristics: a literature review," Los Alamos National Laboratory Report LA-13070-MS, 1996.

[13] B. R. Ellis and A. J. Bougard, "Dynamic testing and stiffness evaluation of a six-storey timber framed building during construction," Engineering Structures, vol. 23, no. 10, pp. 1232-1242, 2001.

[14] Z. Wang, W. Yan, and Y. Shao, “Three-step method for stiffness identification of inter-story shearing structures under ambient excitation," Tsinghua Science and Technology, vol. 14, no. 2, pp. 69-74, 2009.

[15] E. Khanmirza, N. Khaji, and V. J. Majd, "Model updating of multistory shear buildings for simultaneous identification of mass, stiffness and damping matrices using two different softcomputing methods," Expert Systems with Applications, vol. 38, no. 5, pp. 5320-5329, 2011.

[16] J. Zhang, Development of a parameter-insensitive artificial immune system for structural health monitoring [M.S. thesis], Worcester Polytechnic Institute, Worcester, Mass, USA, 2014. 

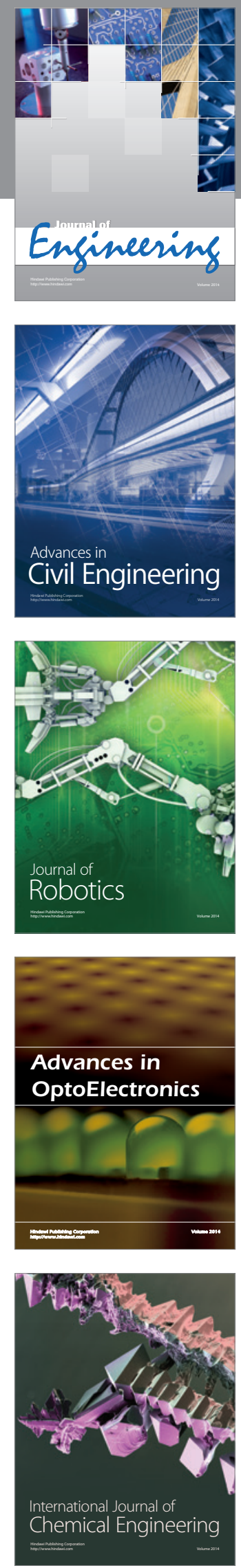

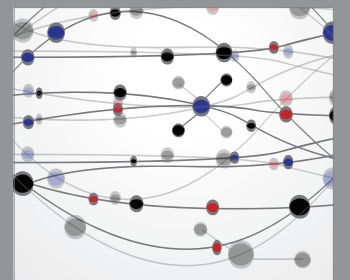

The Scientific World Journal
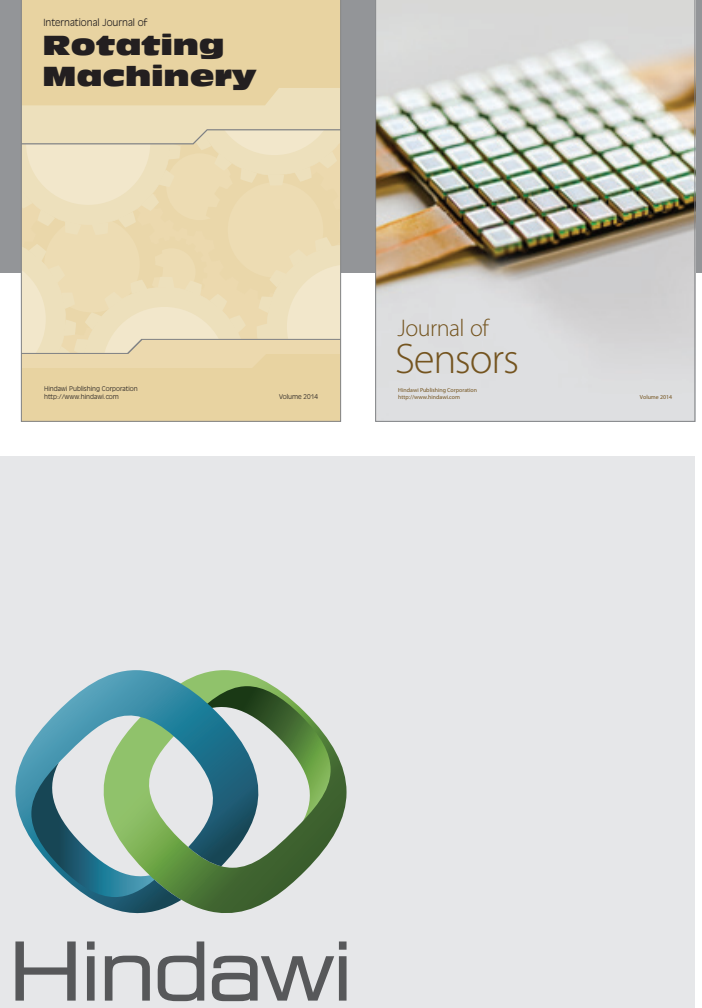

Submit your manuscripts at http://www.hindawi.com
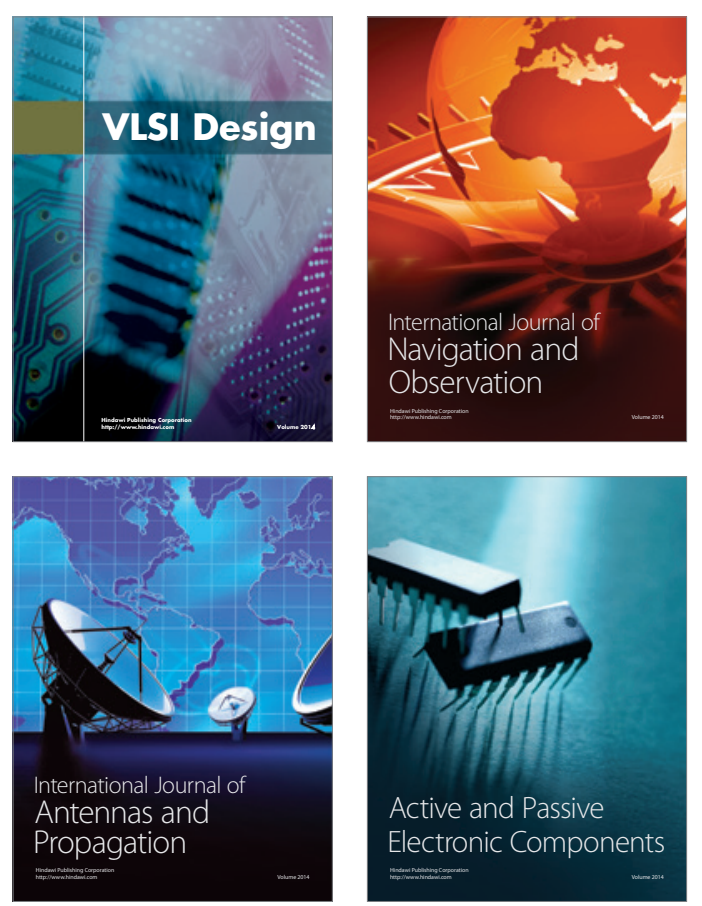
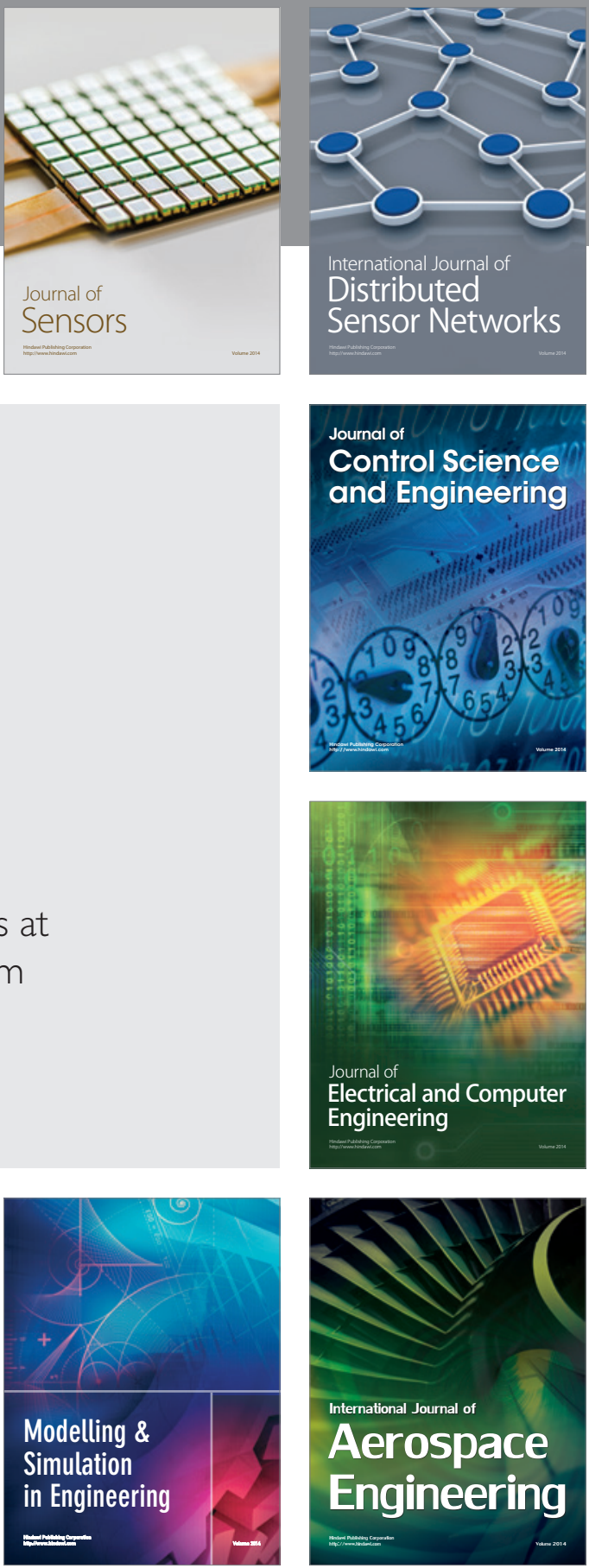

Journal of

Control Science

and Engineering
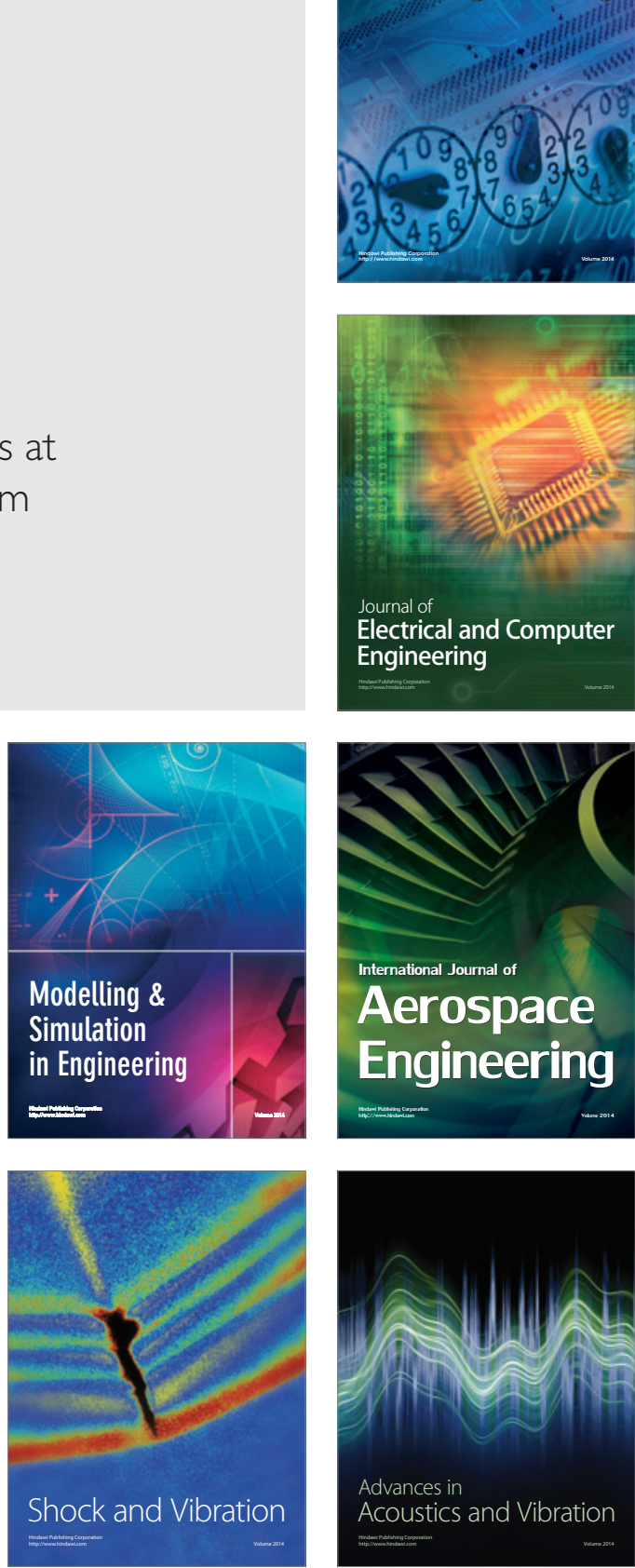